\title{
Optimal design of an horizontal axis wind turbine using blade element momentum theory
}

\author{
Jinane Radi ${ }^{1 *}$, Abdelouahed Djebli ${ }^{1}$ \\ ${ }^{1}$ Department of physics, Energetic Laboratory, Sciences Faculty AEU,Tetouan, Tetouan 93030,Morocco
}

\begin{abstract}
The aerodynamic modelling of the wind turbine blades is a vital step in the design of the turbine. Several design methods are available for the aerodynamic design of the rotor,however,in this study a mathematical model based on blade element momentum concept is applied. the purpose of this work is to optimize the distribution of chord and twist angle along the blade span of a 20 KW HAWT using the BEM method, the blade design parameters such as the optimum lift and drag coefficient, chord and twist angle, the axial induction factor(a) and the angular induction factor(a') of the designed HAWT are codified and estimated using the MATLAB software. The results of the analysis show on the one hand a decrease in chord length and twist angle along the blade length and on the other hand the maximum values that can be achieved by the axial and angular inductions factors are respectively 0.3325 and 0.175 . This approach can be effectively implemented for the analysis of HAWTs operating at different characteristics of the designed blade.
\end{abstract}

Keywords: BEM method, S809 airfoil, Horizontal axis wind turbine, Tip speed ratio, chord and twist distribution, aerodynamic study, inductions factors.

\section{Introduction}

The increasing concerns with environmental and economic issues are driving the search for more durable electrical sources. Wind energy on the other hand, has proven to be the most popular source of clean energy for regions where wind speed meets ideal magnitude and stability. this renewable energy source can be relied on in the longer time. the lack of harmful emissions is the foremost significant benefit of wind energy electricity production. This type of energy is currently experiencing rapid growth widely seen as a serious alternative to combat the greenhouse effect. Wind turbines come in a variety of shapes and sizes, they can be mainly classified into horizontal axis wind turbine (HAWT) and vertical axis wind turbine (VAWT). Today, the HAWT is the much more prevalent form of wind turbine, and the type which is the primary focus of this article. [1]

According to experience the major characteristics of wind turbine performance are based on the aerodynamic forces generated by the mean wind.[2] the design of an aerogenerator is essentially based on aerodynamic modelling, the dimensioning of this geometrical form has a high impact on its energy efficiency and, as a result on its economic profitability. Aerodynamic optimization has widely become an issue of considerable focus to figure out the geometry of an aerodynamic configuration within certain design limitations [3].the aim of optimization is to get the highest possible aerodynamic efficiency at a single design wind speed.

Various computational tools have been used to design wind turbine blades. Computational Fluid Dynamic (CFD) is frequently utilized as a design process, which produces highly accurate results. On the alternative hand, longer time for calculation and big informatics memory is needed. The mathematical model based on the BEM theory which based on Glauret theory is most frequently used in industry and scientific research due to its mid-fidelity results but with less time expenses.[4,5]

The tip loss correction is one of the genuinely significant BEM adjustments. The notion of a tip loss was introduced by Prandtl's to simplify the wake of the turbine by modelling the helical vortex wake pattern as vortex sheets generated by the mean flow but having no direct impact on the wake itself.[6]

In this project our study was focused on modelling and designing of an HAWT with known blade shape and airfoil characteristics using BEM theory, the blade geometry parameters that include Prandtl's tip loss correction are calculated with the aid of MATLAB.

Finally, our work is structed as follows. Section 2 introduce the concept of airfoils for wind turbine, followed by a section on the mathematical modelling where the BEM theory is developed and used to calculate the optimum parameters of the blade designed. Results and discussion have been discussed next, and the last section covers the conclusion.

\footnotetext{
*Corresponding author: jinaneradi195@gmail.com
} 


\section{Airfoils for wind turbines}

Horizontal axis wind turbine designs use airfoil with a particular geometric shape to convert the kinetic energy in the wind into valuable energy. The wind turbine blades cross sections take the form of airfoils. The expected aerodynamic performance, maximum desired rotor power, estimated airfoil parameters, and strength considerations all influence the width and length of the blade. Several studies and investigations have been performed over the years to design and improve the airfoil performance for wind turbine blades. Some of the most well-known airfoil designers are: The NREL airfoils, The Risø airfoils, CAS-W1 Airfoils, Martin Hepperle, MH airfoils, Delft airfoils. [7,8].

The airfoil behavior of the wind turbine can be divided into three flow domains, such as the attached flow reign, the high lift/stall development reign and the flat plate fully stalled reign. [1,2].

\subsection{Selected airfoil characteristics}

The airfoil profile treated in this study belongs to S809. The S809, a 21\% thick laminar-flow airfoil, has been designed, analysed theoretically, and practically validated for HAWT applications in the low-turbulence wind tunnel of the Delft University of Technology Low Speed Laboratory, The Netherlands.

Comparisons to comparable airfoils illustrate the fixed maximum lift coefficient as well as the lower profiledrag coefficients, indicating that the major objectives have been achieved.[8]. This choice is also supported by the ease at which study results can be accessed. Figure 1 shows the shape of the S809 profile.

S809 Airfoil

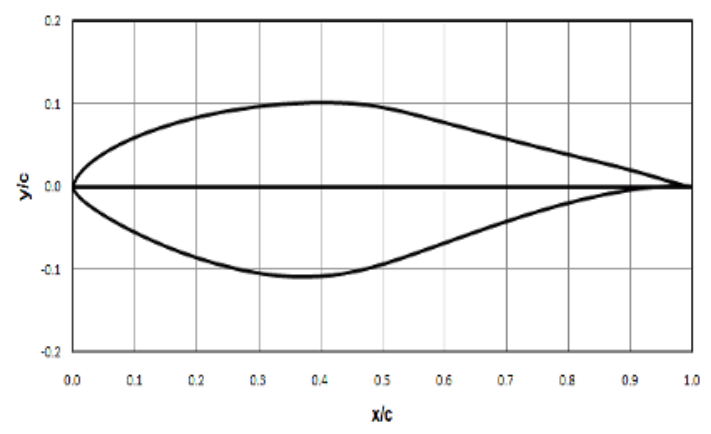

Fig. 1. S809 airfoil.

The lift and drag coefficients for the S809, are graphically represented in figure 2 . As the angle of attack increases, so does the lift coefficient, up to 16 degrees, as shown by this graph. In this angle range a phenomenon recognize as stall occurs where there is a massive rise in drag and a sharp reduction in lift. A rapid increase in the stall angle is also shown by the drag coefficient curve.

These results were in line with the experimental data.

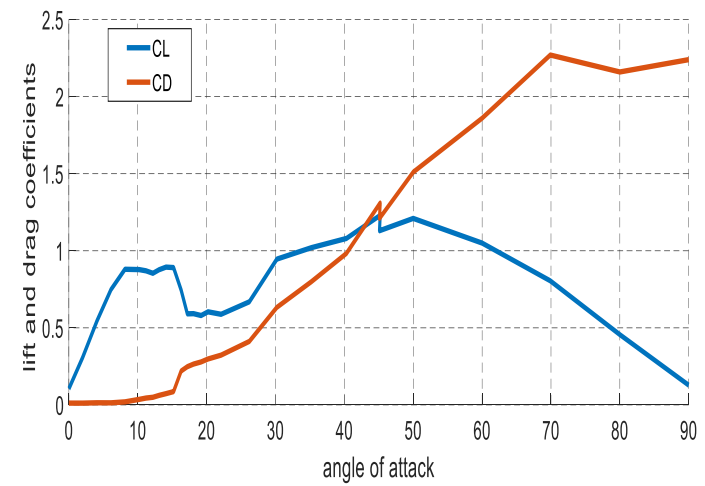

Fig .2. Lift and drag coefficients for the S809 airfoil.

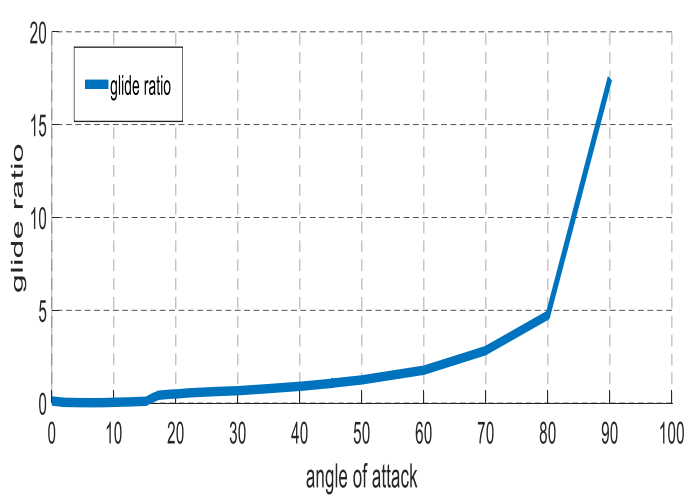

Fig. 3. glide ratio for S809 airfoil.

Also the change of glide ratio of the S809 with different angle of attacks is graphically illustrated in Figure 3 , as it is stated before, as airfoil drag increases, there is a clear decrease in the maximum attainable power. Since it clearly benefits the blade designer to use or design airfoils with low glide ratio, $\alpha$ and $\mathrm{CL}$ values which corresponds to the minimum glide ratio is chosen as $\alpha$ design and CL, design using airfoil data.

\section{Mathematical Modelling}

\subsection{The blade element momentum theory}

The BEM model, commonly known as strip theory, was first suggested by Glauret and is utilized to design wind turbine rotors. This approach is built on two aerodynamic theories, accepting several simplifications The first theory is the axial momentum theory which uses a one-dimensional model, the second is the blade element theory, which takes the rotation of the air into consideration. To determine all of the parameters required to dimension the blades, a combination of the two procedures is required. 
The blade is expected to be divided into $\mathrm{N}$ parts in order to apply blade element momentum analysis showed in figure 4. The analysis is built on certain assumptions, such as that there is no aerodynamic interaction between different blade elements and the forces on the blade elements are determined by the lift and drag coefficients $[9,10]$.

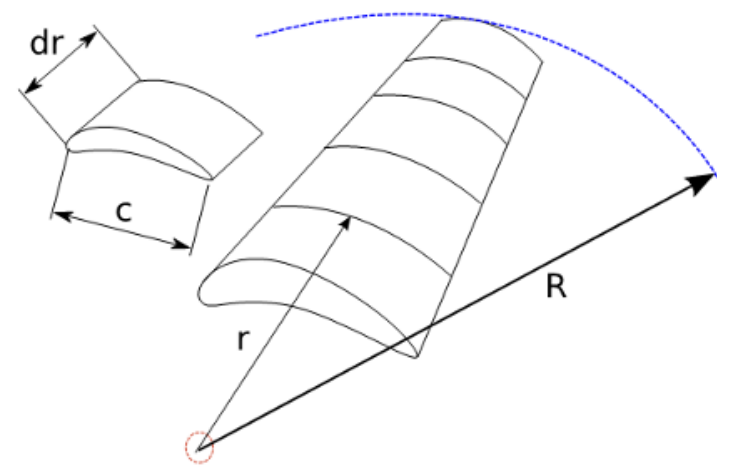

Fig. 4. Blade element model.

Figure 5 shows the velocities and forces on a blade element of a wind turbine blade. As a result, we get the following equations:

$$
\begin{aligned}
d T & =\rho V_{\infty}^{2} 4 a(1-a) \pi r d r \\
d M & =\rho V_{\infty} w 4 a^{\prime}(1-a) \pi r^{3} d r
\end{aligned}
$$

Where $\rho$ is the air density , $\mathrm{r}$ is the local radius, $\mathrm{w}$ is the angular velocity of the turbine, $V_{\infty}$ is the free stream velocity , a is the axial induction factor ,a' is the tangential induction factor.

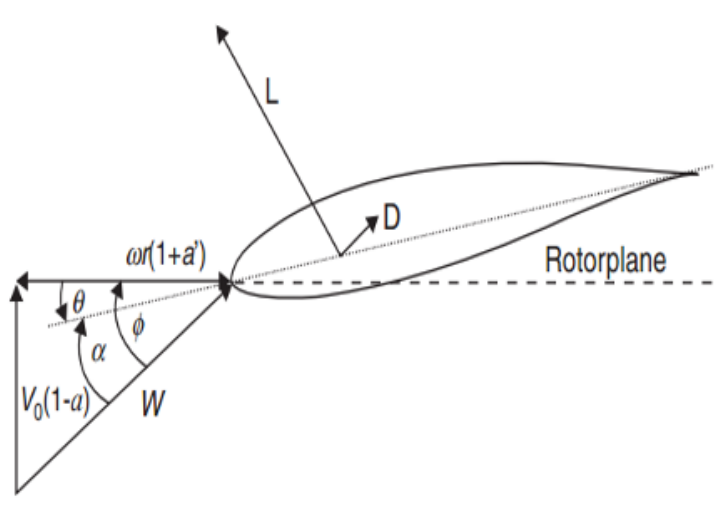

Fig. 5. Blade section for a given radius.

According to the blade element theory and by referring to figure, the lift coefficient can be expressed by:

$$
C_{L}=\frac{d L}{d q}
$$

And the drag coefficient

$$
C_{D}=\frac{d D}{d q}
$$

With $\quad d q=\frac{1}{2} \rho W^{2} C_{r} d r$

Also, the thrust and normal coefficients can be written respectively as

$$
\begin{gathered}
C_{t}=\frac{d F_{t}}{d q} \\
C_{n}=\frac{d F_{n}}{d q}
\end{gathered}
$$

With $C_{n}$ and $C_{t}$ are respectively the normal and tangential loads coefficients :

$$
\begin{aligned}
& C_{n}=C_{l} \cos \phi+C_{d} \sin \phi \\
& C_{t}=C_{l} \sin \phi-C_{d} \cos \phi
\end{aligned}
$$

The axial force $\mathrm{dT}$ and angular torque $\mathrm{dM}$ for a blade element are:

$$
\begin{aligned}
& d T=B C_{t} d q=\frac{1}{2} \rho N_{b} C_{n} \frac{V_{\infty}^{2}(1-a)^{2}}{\sin \phi^{2}} C_{r} \mathrm{dr} \\
& d M=B C_{t} d q=\frac{1}{2} \rho N_{b} C_{t} \frac{V_{\infty}^{2}(1-a)^{2}}{\sin \phi^{2}} C_{r} \mathrm{dr}
\end{aligned}
$$

Now, if one substitutes the left sides of equations (1) and (2) with the right sides of equations (10) and (11), respectively, the factors of the induced velocity can be obtained as

$$
\begin{aligned}
a & =\frac{\sigma C_{n}}{4 \sin \phi^{2}+\sigma C_{n}} \\
a^{\prime} & =\frac{\sigma C_{t}}{4 \sin \phi \cos \phi-\sigma C_{t}}
\end{aligned}
$$

Where $\sigma$ is the solidity of rotor defined as:

$$
\sigma=\frac{N_{b} C_{r}}{2 \pi r}
$$

And as well the thrust coefficient is defined as:

$$
C_{T}=4 a(1-a)
$$

Also the power coefficient can be represented as follow

$$
C_{P}=\frac{8}{\lambda^{2}} \int_{\lambda_{h}}^{\lambda} \lambda_{r}^{3} a^{\prime}(1-a)\left(1-\frac{C_{D}}{C_{L}} \cot \phi\right) d \lambda_{r}
$$

Where $\lambda_{h}$ is the local tip speed ratio at the hub

\subsection{Prandtl's loss factor correction}

Since the suction side of a blade has lower pressure than the pressure side, air circulates around the tip from the lower to upper surface, decreasing lift and therefore power production close to the tip. The impact is most apparent with fewer, wider blades. A number of methods have been suggested for including the impact of the tip loss. The correction that will be taken into account during this study is tip loss correction with a restricted number of blades. This phenomenon is quantified by the traffic reduction factor proposed by Prandtl and outlined by the following formula:

$$
F=\frac{2}{\pi} \cos ^{-1}\left\{\left[\frac{-\left(\frac{N_{b}}{2}\right)\left[1-\frac{r}{R}\right]}{\left(\frac{r}{R}\right) \sin \left(\phi_{o p t}\right)}\right]\right\}
$$


After considering tip loss factor equations (12), (13)

(15)and (16) should be changed to:

$$
\begin{gathered}
\boldsymbol{a}=\frac{1}{\frac{4 F \sin \phi^{2}}{\sigma C_{\boldsymbol{a}}}+1} \\
\boldsymbol{a}^{\prime}=\frac{1}{\frac{4 F \sin \phi \cos \phi}{\sigma C_{\boldsymbol{t}}}-1} \\
C_{T}=4 a F(1-a) \\
C_{P}=\frac{8}{\lambda^{2}} \int_{\lambda_{h}}^{\lambda} \lambda_{r}^{3} F a^{\prime}(1-a)\left(1-\frac{C_{D}}{C_{L}} \cot \phi\right) d \lambda_{r}(21)
\end{gathered}
$$

\subsection{Blade design procedure}

The aerodynamic design of optimum rotor blades from a well - known airfoil type mean determining the geometric parameters such as chord length distribution and twist distribution along the blade length for a certain tip speed ratio at which the power coefficient of the rotor is maximum. By dividing the blade length into $\mathrm{N}$ elements, the local tip speed ratio for each blade element can be computed.

$$
\lambda_{r}=\lambda\left(\frac{\mathrm{r}}{R}\right)
$$

The equation for optimal local oncoming airflow angle at radial distance $r$ is:

$$
\phi_{\text {opt }}=\frac{2}{3} \tan ^{-1}\left(\frac{1}{\lambda_{r}}\right)
$$

Chord length distribution can be calculated for each blade element using equation (24)

$$
C_{r}=\frac{8 \pi r}{N_{b} C_{L d e s}}(1-\cos \phi)
$$

Where $C_{L \text { des }}$ is chosen such that the glide ratio is minimum at each blade element.

The twist distribution can easily be determined by using equation (25)

$$
\theta=\phi_{\text {opt }}-\alpha_{\text {design }}
$$

Where $\alpha_{\text {design }}$ is the design angle of attack at which $\mathrm{CL}$ is obtained.

\section{Results and Discussion}

The optimization of wind turbine was performed by a MATLAB code, which uses BEM analysis. Different parameters were utilized in the BEM analysis which might be entered in optimization process, these parameters include turbine diameter, chord length, angle of attack, airfoil type, number of blades. Table 1 shows the elements of the rotor wind turbine that was applied.

\begin{tabular}{|l|l|}
\hline Wind turbine type & $\begin{array}{l}\text { Horizontal axis wind } \\
\text { turbine }\end{array}$ \\
\hline Profile type & S809 \\
\hline Rotational speed & $120 \mathrm{rpm}$ \\
\hline Number of blades & 3 \\
\hline Rated power & $20 \mathrm{kw}$ \\
\hline Wind speed & $10 \mathrm{~m} . \mathrm{s}-1$ \\
\hline Rotor Diameter & $10 \mathrm{~m}$ \\
\hline Swept area & $75.4 \mathrm{~m} 2$ \\
\hline Reynolds number & 300000 \\
\hline CL_design & 0.748 \\
\hline design angle of attack & 16 degrees \\
\hline
\end{tabular}

Table 1. characteristics of the designed blades

The results of the optimal chord length and twist angle distributions are summarized in the graphs below. Figure 6 illustrates the optimum chord length of the airfoil S809 as a function of the blade length with and without the tip hub loss factor. The curve is plotted using the ratio $\mathrm{r} / \mathrm{R}$ in $\mathrm{x}$-axis and chord length in the $\mathrm{y}$ axis. The chord length decreases as the radial distance increases, as shown by the curve. 


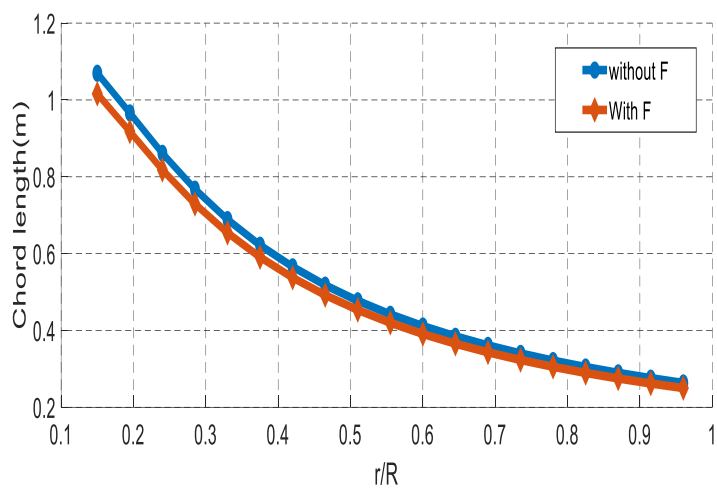

Fig. 6. Chord length distribution of the designed blade.

Twist angle distribution as a function of the blade length is represented in figure 7. The curve is plotted using the ratio $r / R$ in $x$ - axis and twist angle in the $y$ axis with and without the tip hub loss factor. The curve shows that as the blade length increases, the chord length decreases.

These results led to the conclusion that the tip-hub loss factor has an effect on the blade chord and twist angle distributions

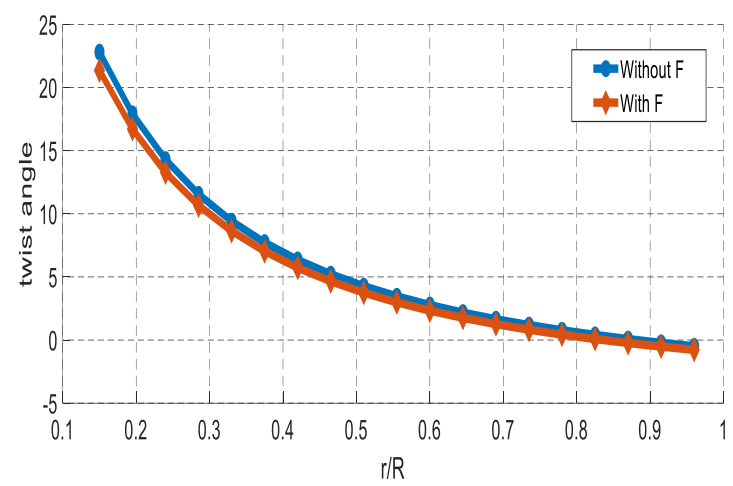

Fig. 7. Twist angle distribution of the designed blade.

The blade design process begins with the assumption of a and a'. As the BEM analysis progresses, the preliminary guesses for the induction factors are replaced with the newly discovered values, and the procedure continues until an optimal solution is identified, as shown in Figures 8 and 9.

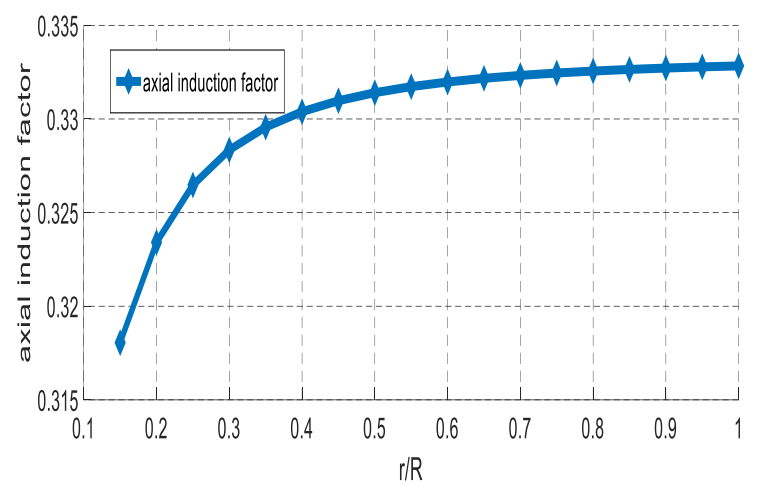

Fig. 8. Axial induction factor.

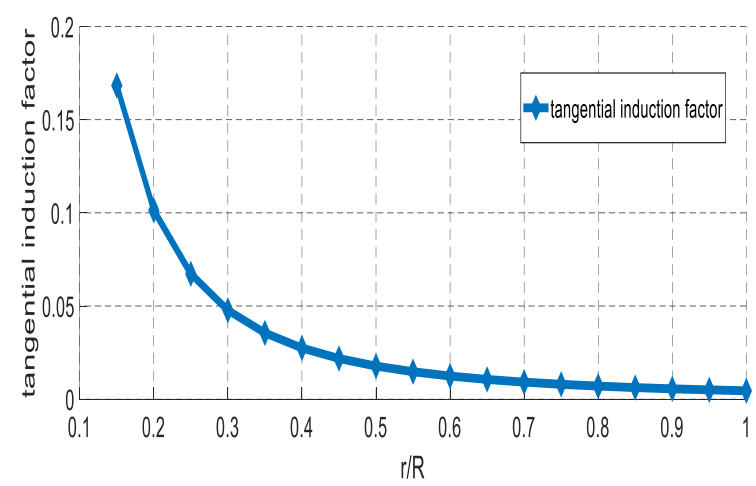

Fig. 9. Tangential induction factor.

\section{Conclusion}

In the present study an attempt has been made to predict the optimal parameters of a $20 \mathrm{~kW}$ HAWT blades using a S809 airfoil, the design parameters have been obtained with the aid of the blade element momentum theory combined with the Prandtl's correction. Chord length and twist angle distributions ,axial induction factor ,tangential induction factor of the designed blade were calculated and programmed through the MATLAB software

The simulation results are quite encouraging and it may be possible to apply this model for further airfoil series. Otherwise, there are other approaches to predict blade performance and to design blades that may be more applicable in some situations. However, further improvements should be implemented to the model to fulfilment the HAWT. 


\section{References:}

1. U. Chaudhary, P. Mondal, P. Tripathy, S. K. Nayak and U. K. Saha,Modeling and optimal design of small HAWT blades for analyzing the starting torque behavior, Eighteenth National Power Systems Conference (NPSC),IEEE,16(2014)

2. J. F. Manwell ,J. G. McGowan, A. L. Rogers, Wind Energy Explained Theory, Design and Application , (2002),John Wiley \& Sons Ltd., West Sussex, Britain

3. B. Bavanish, K. Thyagarajan, Optimization of power coefficient on a horizontal axis wind turbine using bem theory. Renewable and Sustainable Energy Reviews, 26, 169182,(2013).

4. Y.El khchine, M. Sriti.Improved blade element momentum theory (BEM) for predicting the aerodynamic performances of horizontal axis wind turbine blade Technische Mechanik , 38, 191- 202 (2018)
5. A.Ozkan, Aerodynamic design of turbine blades using full dynamic,(2013), in Proceeding of the conference of Wind Energy Science and technology October 3-4, Ankara, Turkey (2013)

6. Y. El khchine, M. Sriti, Tip loss factor effects on aerodynamic performances of horizontal axis wind turbine,in Proceeding of the International Conference on Advances on Clean Energy Research, ICACER, Berlin, Germany, 118,136140 (2017)

7. J. F. Manwell ,J. G. McGowan, A. L. Rogers, Wind Energy Explained Theory, Design and Application , John Wiley \& Sons Ltd., West Sussex, Britain (2002)

8. M.Somers, Design and experimental results for the S809 airfoil, united states (1997)

9. W.Wang, S.Caro, , F.Bennis, 0.R.Salinas Mejia ,A Simplified Morphing Blade for Horizontal Axis Wind Turbines. Journal of Solar Energy Engineering, 136.(2013)

10. Z.L.Mahri, M.S.Rouabah,S.Zid,Calcul des efforts aérodynamiques agissant sur les pales d'une petite eolienne,Revue des energies renouvelables ,10 241-256 (2007). 